\title{
Increased glyceraldehyde-3-phosphate dehydrogenase expression indicates higher survival rates in male patients with hepatitis $B$ virus-accociated hepatocellular carcinoma and cirrhosis
}

\author{
SHUANG LIU ${ }^{1 *}$, PENGFEI ZHU ${ }^{2 *}$, LING ZHANG ${ }^{3}$, ZHUO LI $^{1}$, QUANJUN LV ${ }^{4}$, \\ SUJUN ZHENG ${ }^{1}$, YANG WANG ${ }^{1}$ and FENGMIN LU ${ }^{2}$
}

\author{
${ }^{1}$ Beijing Artificial Liver Treatment and Training Center, Beijing Youan Hospital, Capital Medical University, Beijing 100069; \\ ${ }^{2}$ Department of Microbiology and Infectious Disease Center, Peking University Health Science Center, Beijing 100086; \\ ${ }^{3}$ Department of Hepatobiliary Surgery, Henan Cancer Hospital, Zhengzhou, Henan 450008; \\ ${ }^{4}$ Department of Nutrition and Food Hygiene, College of Public Health, \\ Zhengzhou University, Zhengzhou, Henan 450052, P.R. China
}

Received May 4, 2014; Accepted January 15, 2015

DOI: $10.3892 / \mathrm{etm} .2015 .2309$

\begin{abstract}
Elevated expression of glyceraldehyde-3-phosphate dehydrogenase (GAPDH) has been reported in different human malignancies. To understand its role in hepatitis $\mathrm{B}$ virus (HBV) infection-associated hepatocellular carcinoma (HCC), the expression of GAPDH was quantitatively measured in a cohort of 72 male HCC patients without preoperative treatment, all with evidence of chronic HBV infection. Using C-terminal banding protein 1 (CTBP1) or hypoxanthine phosphoribosyltransferase 1 (HPRT1) as reference genes, the level of GAPDH mRNA in tumor tissue was found to be significantly higher compared with that in paired non tumor tissues ( $\mathrm{P}=0.0087$ for CTBP1; $\mathrm{P}=0.0116$ for HPRT1). Accordingly, compared with the non-tumor tissue, $37.5 \%$ (27/72) of patients' tumor tissues had a more than 2-fold increase of GAPDH
\end{abstract}

Correspondence to: Professor Fengmin Lu, Department of Microbiology and Infectious Disease Center, Peking University Health Science Center, 38 Xueyuan Road, Haidian, Beijing 100086, P.R. China

E-mail: lu.fengmin@hsc.pku.edu.cn

Dr Ling Zhang, Department of Hepatobiliary Surgery, Henan Cancer Hospital, 127 Dongmin Road, Zhengzhou, Henan 450008, P.R. China

E-mail: zhanglily56@126.com

${ }^{*}$ Contributed equally

Abbreviation: $\mathrm{HBV}$, hepatitis $\mathrm{B}$ virus; $\mathrm{HCC}$, hepatocellular carcinoma; GAPDH, glyceraldehyde-3-phosphate dehydrogenase; RT-qPCR, reverse transcription-quantitative polymerase chain reaction; CTBP1,C-terminal banding protein 1; HPRT1, hypoxanthine phosphoribosyltransferase 1; araC, cytosine arabinoside; MTT, 3-(4,5-dimethylthiazol-2-yl)-2,5-diphenyltetrazolium

Key words: hepatocellular carcinoma, glyceraldehyde-3-phosphate dehydrogenase, hepatitis B virus, liver cirrhosis, chemotherapy expression. Furthermore, following knockdown GAPDH expression via siRNA transient transfection, HepG2 cells exhibited enhanced resistance to cytosine arabinoside ( $\mathrm{IC}_{50}$, $308.28 \mu \mathrm{M}$ vs. $67.68 \mu \mathrm{M}$ in the control; $\mathrm{P}=0.01)$. Notably, higher GAPDH expression was significantly associated with lower liver fibrosis score $(\mathrm{P}=0.0394)$ and a tendency towards higher survival rates for patients with HCC. To the best of our knowledge, the present study is the first study to report that the elevated expression levels of GAPDH in HCC tumor tissue may be relevant to an improved fibrosis score and survival probability in male patients with HBV infection; however, the underlying mechanism requires further investigation.

\section{Introduction}

Hepatocellular carcinoma (HCC) is the third leading cause of cancer-associated mortality in males and the sixth leading cause in females worldwide (1). In addition, HCC is ranked as the second leading cause of cancer-related mortality in China (2). Over $50 \%$ of the annual HCC cases reported worldwide are diagnosed in the Chinese population (3), while hepatitis $\mathrm{B}$ virus (HBV) infection is responsible for $80 \%$ of all HCC causes in China (4). While a number of studies have revealed that $\mathrm{HBV}$ infection is the key pathogenic factor for the development of HCC, male gender and cirrhosis have also been found to be independent risk factors for the occurrence of HCC (5-7). However, few studies exist on patients presenting all these risk factors.

Glyceraldehyde-3-phosphate dehydrogenase (GAPDH) was initially considered to be an essential glycolytic enzyme that is expressed in all prokaryotic and eukaryotic organisms. GADPH plays a major role in cellular metabolism, converting glyceraldehyde-3-phosphate to 1,3-diphosphoglycerate (8). In addition, studies have demonstrated that GAPDH is involved in certain important physiological functions, including the transport of transfer RNA (9), translational control (10) and binding with viral RNAs $(11,12)$. Furthermore, increased GAPDH expression has been previously reported in renal 
cell carcinoma (13), lung cancer (14), breast cancer (15), prostate carcinoma (16) and HCC (17). A number of studies have demonstrated that the elevated expression of GAPDH is correlated with chemotherapy-induced DNA damage response $(18,19)$. The induction of cell cycle arrest in p53-proficient carcinoma cells through GAPDH abrogation indicates that GAPDH-depleting agents may have a cytostatic effect in cancer cells (20). In addition, higher intranuclear GAPDH expression has been found to be correlated with higher cell sensitivity to mercaptopurine treatment in human leukemia cell lines (21). Therefore, investigating the underlying mechanism resulting in aberrant expression of GAPDH in the development of $\mathrm{HCC}$ in patients presenting the aforementioned risk factors is essential.

Reverse transcription-quantitative polymerase chain reaction (RT-qPCR) is a powerful tool used to detect the level of gene expression in certain tissues under normal and disease conditions $(22,23)$. The authors of the present study have previously used RT-qPCR to analyze the stability of six candidate genes in paired tumor and non-tumor tissues from 33 male with untreated HBV-associated HCC and cirrhosis, using the geNorm and NormFinder software (24). C-terminal banding protein 1 (CTBP1) and hypoxanthine phosphoribosyltransferase 1 (HPRT1) were demonstrated to be evidently more stable compared with GAPDH, while CTBP1 was found to have the most stable gene expression (24). The present study aimed to investigate whether GAPDH expression was aberrantly altered in the tumors of male HCC patients with chronic HBV infection. The clinical significance was also addressed.

\section{Materials and methods}

Patient information and sample collection. HCC tumor and paired non-tumor tissue samples were obtained from 72 untreated male patients, suffering from HBV-associated HCC with cirrhosis. The patients underwent hepatectomy at the Henan Cancer Hospital (Zhengzhou, China) between July 2009 and October 2010. The selected HCC patients met all the following criteria: seropositive for HBV surface antigen (HBsAg) or HBV DNA-positive in tumor tissues; received no chemotherapy prior to surgery; male gender; and suffered from liver cirrhosis. Liver cirrhosis and HCC were evaluated by two pathologists independently. The Ishak scoring system was used to assess fibrosis stage (25). Samples were collected from the tumor and paired non-tumor tissues following hepatectomy and the specimens were snap-frozen in liquid nitrogen. The age range of the 72 patients was 34-76 years (mean age, $51.25 \pm 9.91$ years). The Institutional Review Board of Peking University (Beijing, China) approved all the procedures in the present study. Written informed consent was obtained from the patients/patients' families prior to their participation.

Analysis of GAPDH mRNA expression levels in tumor and non-tumor tissues from HCC patients using RT-qPCR. The tissue specimens were ground in liquid nitrogen and homogenized in TRIzol (Invitrogen Life Technologies, Carlsbad, CA, USA) using a mortar. Total RNA was extracted with TRIzol (Invitrogen Life Technologies) according to the manufacturer's instructions. Genomic DNA contamination was removed by on-column digestion using the RNase-free DNase kit (Takara
Bio Inc., Otsu, Japan). The concentration of the isolated total RNA was calculated by measuring the absorbance (A) at 260 and $280 \mathrm{~nm}$ using a NanoDrop ND-2000 spectrophotometer (Thermo Fisher Scientific Inc., Wilmington, DE, USA). A260/A280 ratio of $>1.90$ and $28 \mathrm{~S} / 18 \mathrm{~S}$ ratio of $\geq 1.7$ were the threshold values for inclusion of the RNA samples in this study. The integrity of the RNA samples was confirmed by electrophoresis on a $1 \%$ agarose gel. First-strand cDNA was synthesized using a random primer and the RevertAid First Strand cDNA Synthesis kit (Fermentas, Vilnius, Lithuania), according to the manufacturer's instructions. The primers used in the RT-qPCR assays of GAPDH were designed using the Primer Premier 5.0 software (Premier Biosoft, Palo Alto, CA, USA). The Roche LightCycler 480 detection system (Roche Diagnostics GmbH, Mannheim, Germany) was used for RT-qPCR analysis. The reactions were performed in a final volume of $20 \mu \mathrm{l}$, containing $10 \mu \mathrm{l}$ of $\mathrm{SYBR}^{\circledR}$ Green master mix (Roche Diagnostics $\mathrm{GmbH}$ ), $0.5 \mu \mathrm{l}$ of each $10 \mu \mathrm{M}$ primer (500 nM), $1 \mu \mathrm{l} \mathrm{cDNA}$ and $8 \mu \mathrm{l}$ nuclease-free sterile water. All the standard solutions and samples were analyzed in triplicate on 96-well reaction plates. The cycling conditions were set as follows: $10 \mathrm{~min}$ template denaturation at $95^{\circ} \mathrm{C}, 40$ cycles of denaturation at $95^{\circ} \mathrm{C}$ for $30 \mathrm{sec}$ and elongation at $72^{\circ} \mathrm{C}$ for $30 \mathrm{sec}$. Melting-curve analysis was performed following RT-qPCR and the baseline and cycle threshold values $(\mathrm{Ct}$ values) were automatically determined for all the plates using the Roche LightCycler 480 software. A Ct value difference between triplicates of $\leq 1$ was considered as acceptable and was used to calculate the average $\mathrm{Ct}$ values. Genes exhibiting an $>2$-fold increase $\left(2^{-\Delta C t}>2\right)$ or an $<0.5$-fold decrease $\left(2^{-\Delta \mathrm{Ct}}<0.5\right)$ in their expression levels were considered to be differentially expressed, whereas genes with an $\leq 2$-fold increase or $\geq 0.5$-fold decrease $\left(0.5 \leq 2^{-\Delta \mathrm{Ct}} \leq 2\right)$ were defined as equally expressed, as previously reported (26).

Construction of GAPDH expression vectors. The $1026 \mathrm{bp}$ GAPDH cDNA containing the entire open reading frame was amplified using RT-qPCR and cloned into the expression vector, pIRES2-EGFP (Clontech Laboratories, Inc., Palo Alto, CA, USA). The GAPDH primers used were as follows: sense, 5'-CCGGAATTCATGGGGAAGGTGAAGG-3'; and antisense, 5'-GACGTCGACTTACTCCTTGGAGGCCATG-3'. The GAPDH-expressing vector, pGAPDH-IRES2-EGFP, was constructed and confirmed by automated sequencing, in order to establish the direction of cloning and determine whether the sequence was correct.

HepG2 and PLC/PRF/5 cell cultures, drug treatment and viability assay. HepG2 cells (American Type Culture Collection, Manassas, VA, USA) were maintained in Dulbecco's modified Eagle's medium supplemented with $10 \%$ fetal bovine serum (Gibco Life Technologies, Carlsbad, CA, USA), while PLC/PRF/5 cells (American Type Culture Collection) were maintained in RPMI 1640 medium supplemented with $10 \%$ fetal bovine serum. In this study, the stock solutions of $10^{4} \mu \mathrm{M}$ epirubicin (Pfizer, New York, NY, USA) or $10^{6} \mu \mathrm{M}$ araC (SigmaAldrich, St. Louis, MO, USA) were used, which were dissolved in phosphate-buffered saline and stored at $-20^{\circ} \mathrm{C}$.

Cell viability was determined using MTT [3-(4,5-dimethylthiazol-2-yl)-2,5-diphenyltetrazolium; Promega Corporation, 
A

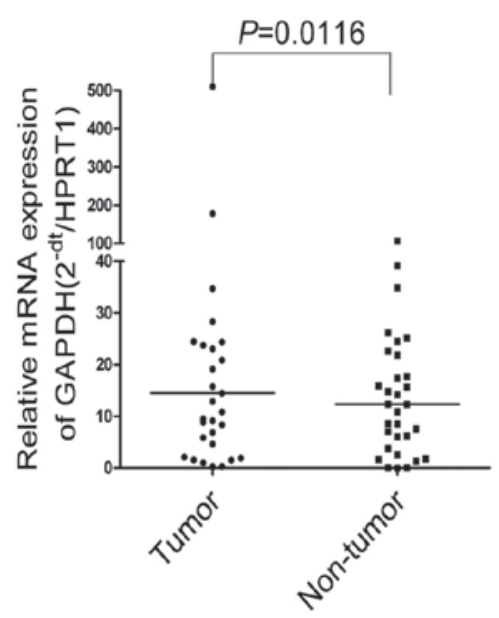

B

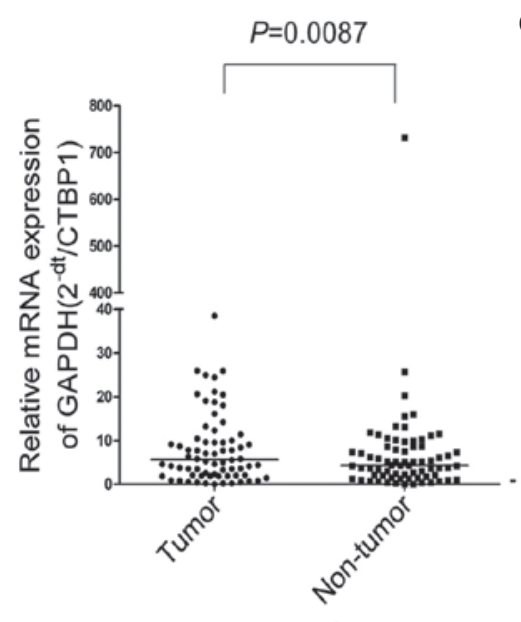

C

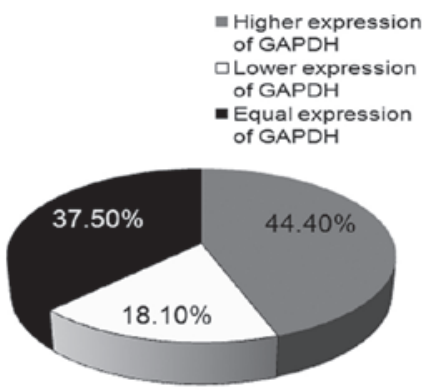

Figure 1. Relative expression levels of GAPDH in paired tumor and non tumor tissues, (A) in 33 patients, using hypoxanthine phosphoribosyltransferase 1 (HPRT1) as the reference gene, and (B) in 72 patients, using C-terminal banding protein 1 (CTBP1) as the reference gene. (C) Distribution of relative GAPDH expression levels in 72 patients. GAPDH, glyceraldehyde-3-phosphate dehydrogenase.

Madison, WI, USA]. HepG2 cells (7,000 cells/well) and $\mathrm{PLC} / \mathrm{PRF} / 5$ cells $(8,000$ cells/well) were plated into 96 -well plates and cultured for two days with various drug concentrations $\left(10^{-3}-10 \mu \mathrm{M}\right.$ epirubicin or $5-5 \times 10^{4} \mu \mathrm{M}$ araC). The $\mathrm{IC}_{50}$ values, representing the half maximal inhibitory concentration, were calculated using the GraphPad Prism 5.0a software (GraphPad Software Inc., San Diego, CA, USA) software. To evaluate the effect of GAPDH expression on drug resistance, the cells were treated with araC or epirubicin at $48 \mathrm{~h}$ after transfection.

The cells were seeded in six-well plates or in 60 -mm dishes and grown for $24 \mathrm{~h}$ before transfection. Plasmids and small interfering RNA (siRNA) molecules were transfected into the cells using Lipofectamine ${ }^{\mathrm{TM}} 2000$ transfection reagent (Invitrogen Life Technologies) according to the manufacturer's instructions. A GAPDH siRNA kit (\#NM-002046) and scrambled control siRNA were purchased from Sigma-Aldrich. The primer sequences used were as follows: GAPDH siRNA sense, 5'-GGUUUACAUGUUCCAAUAUdTdT-3', and anti-sense, 5'-AUAUUGGAACAUGUAAACCdTdT-3'; siRNA negative control sense, 5'-UUCUCCGAACGUGUCACGUTT-3', and anti-sense, 5'-ACGUGACACGUUCGGAGAATT-3'. Initially, western blot analysis was performed to validate the expression of GAPDH in the transfected plasmid (27).

The primary antibodies used in the present study included mouse p21 [MBL(K0081-3); 1:500; Santa Cruz Biotechnology, Inc., Dalla, TX, USA], mouse GAPDH [MBL(M171-3); 1:3,000 Santa Cruz Biotechnology, Inc.] and rabbit $\beta$-actin (sc-1616; 1:1,000; Santa Cruz Biotechnology, Inc.). The cultured cells were collected and the proteins were lysed in RIPA buffer (Applygen Technologies Inc., Beijing, China). Protein samples (30 $\mu \mathrm{g}$ each) were loaded onto $12 \%$ sodium dodecyl sulfatepolyacrylamide gels, electrophoresed and transferred onto nitrocellulose membranes (Amersham Biosciences, Uppsala, Sweden). Briefly, membranes were blocked with $5 \%$ dried milk in phosphate-buffered saline (PBS) for $2 \mathrm{~h}$ followed by incubation with the primary antibodies for $2 \mathrm{~h}$. After 3 washes with PBS containing 0.1\% Tween-20, the membranes were incubated with the IRDye ${ }^{\circledast} 680$ goat anti-rabbit
(\#926-32221; 1:8,000; LI-COR Biosciences, Cambridge, UK) and IRDye ${ }^{\circledR} 680$ goat anti-mouse (\#926-32220; 1:8,000; LI-COR Biosciences) secondary antibodies for $1 \mathrm{~h}$ at room temperature. Protein-antibody complexes were visualized using the secondary antibodies conjugated with Cy5.5 (Amersham-Pharmacia Biotech, Piscataway, NJ, USA) and the LI-COR Odyssey ${ }^{\circledR}$ IR Imaging system (LI-COR Biosciences). Subsequently, siRNA-transfected cells were treated with araC or epirubicin at $48 \mathrm{~h}$ after transfection and MTT assay was performed to measure the cell viability.

Statistical analyses. Student's t-test or Wilcoxon signed-rank test were used for statistical analyses with the SAS software (version 9.1; SAS Institute Inc., Cary, NC, USA). A Kaplan-Meier survival curve was generated to analyze the patients' survival rates following surgery. $\mathrm{P} \leq 0.05$ was considered to indicate a statistically significant difference. The results are expressed the mean \pm standard error of mean of three independent experiments.

\section{Results}

Significantly increased GAPDH expression levels in tumor tissues when compared with non-tumor tissues. Using the HPRT1 housekeeping gene as a reference gene, the mRNA expression levels of GAPDH were detected in 33 out of the 72 cases. Compared with non-tumor tissues, significantly higher GAPDH expression was observed in the tumor tissues $(\mathrm{P}=0.0116$; Fig. 1A). In order to validate this observation in the 72 patients with HCC, CTBP1 was used as the reference gene, since it has been previously demonstrated to be the most stable gene among six candidate housekeeping genes (24). The relative expression of GAPDH was measured in the 72 HCC cases using RT-qPCR. The results of the Wilcoxon signed-rank test demonstrated that GAPDH expression was significantly increased in the HCC tumor tissues when compared with the non-tumor tissues ( $\mathrm{P}=0.0087$; Fig. 1B). In total, $37.5 \%$ of cases (27/72 patients) presented increased expression of GAPDH $\left(2^{-\Delta C t}>2\right), 18.1 \%$ of cases (13/72 patients) presented 
Table I. Patient characteristics according to the expression levels of GAPDH in tumor tissues.

\begin{tabular}{|c|c|c|c|}
\hline Characteristics & $\begin{array}{l}\text { Increased group } \\
\qquad(\mathrm{n}=27), \mathrm{n}\end{array}$ & $\begin{array}{l}\text { Non-increased group } \\
\qquad(\mathrm{n}=45), \mathrm{n}\end{array}$ & P-value \\
\hline Age & & & 0.9036 \\
\hline$<50$ years & 13 & 21 & \\
\hline$\geq 50$ years & 14 & 24 & \\
\hline Abdominal dropsy & & & 0.9374 \\
\hline Yes & 5 & 8 & \\
\hline No & 22 & 37 & \\
\hline Portal vein cancerous thrombus & & & 0.5118 \\
\hline Complete & 7 & 15 & \\
\hline Incomplete & 20 & 30 & \\
\hline Preoperative AFP value & & & 0.7873 \\
\hline$\leq 20 \mu \mathrm{g} / 1$ & 7 & 13 & \\
\hline$>20 \mu \mathrm{g} / 1$ & 20 & 32 & \\
\hline Liver cirrhosis & & & 0.0394 \\
\hline I-III & $21(46.7 \%)$ & 24 & \\
\hline IV-VI & $6(22.2 \%)$ & 21 & \\
\hline
\end{tabular}

Liver fibrosis score was evaluated by two pathologists independently and the Ishak scoring system was used. Higher expression of GAPDH was significantly associated with a lower liver fibrosis score ( $\mathrm{P}=0.0394)$. AFP, $\alpha$-fetoprotein; GAPDH, glyceraldehyde-3-phosphate dehydrogenase .

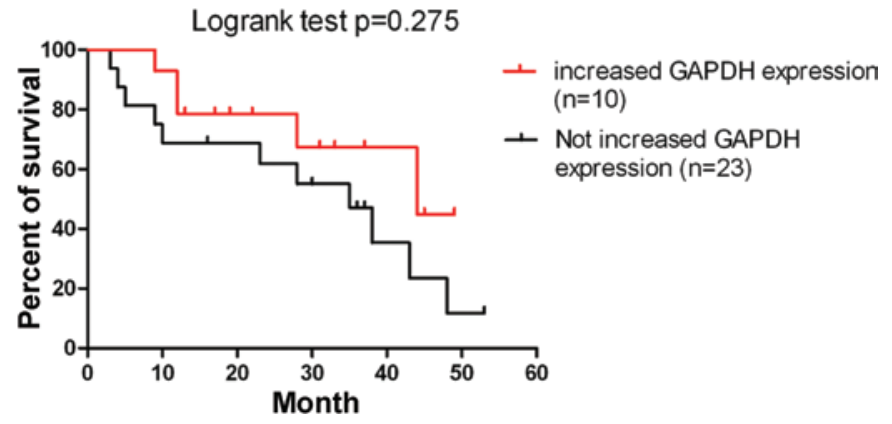

Figure 2. Survival rates (\%) of HCC patients with increased or non-increased GAPDH expression. A clear tendency for higher survival rates of the HCC patients is observed in the increased GAPDH expression group; however, the difference was not statistically significant $(\mathrm{P}=0.275)$. GAPDH, glyceraldehyde-3-phosphate dehydrogenase.

reduced expression of GAPDH $\left(2^{-\Delta \mathrm{Ct}}<0.5\right)$ and $44.4 \%$ of cases (32/72 patients) presented unchanged expression of GAPDH $\left(0.5 \leq 2^{-\Delta \mathrm{Ct}} \leq 2\right)$. Fig. $1 \mathrm{C}$ shows the distribution of the different expression levels of GAPDH detected in the HCC patients.

In order to investigate whether there was an association between the mRNA levels of GAPDH and the clinical characteristics, the patients were further divided into the increased $(n=27)$ and non-increased expression group $(n=45)$, which included the cases presenting reduced and unchanged expression levels of GAPDH. Statistical analysis revealed that the increased expression group was associated with a lower score of liver fibrosis (Ishak score I-III vs. IV-VI; 46.7\%, vs. 22.2\%; $\mathrm{P}=0.0394$; Table I) (25). This indicated that increased GAPDH expression was significantly associated with a lower liver fibrosis score. However, other clinicopathological characteristics, including age, abdominal dropsy, preoperative $\alpha$-fetoprotein value and portal vein cancerous thrombus, did not induce a statistically significantly difference on the expression of GAPDH $(\mathrm{P}>0.05$; Table I).

To investigate whether an association exists between the elevated GAPDH expression and patient survival rates, Kaplan-Meier survival analysis was conducted and patients with increased GAPDH expression presented higher overall survival rates; however, no statistically significant difference was observed ( $\mathrm{P}=0.275$; Fig. 2).

Knockdown of GAPDH expression results in reduced cell sensitivity to chemotherapy with araC in HepG2 cells, but not in PLC/PRF/5 cells. HepG2 and PLC/PRF/5 cells were initially transiently transfected with GAPDH siRNA (siGAPDH) or siRNA NC. Subsequently, the sensitivity of each cell line to araC or epirubicin was assayed individually. Notably, GAPDH expression knockdown was found to significantly alter the HepG2 cell chemotherapy sensitivity to $\operatorname{araC}(308.28 \mu \mathrm{M}$ in the siGAPDH group, vs. $67.68 \mu \mathrm{M} \mu \mathrm{M}$ in the siRNA NC group; $\mathrm{P}=0.01$ ), but not to epirubicin $(10.37 \mu \mathrm{M}$ in the siGAPDH group, vs. $8.96 \mu \mathrm{M}$ in the siRNA NC group). However, GAPDH knockdown did not significantly alter the PLC/PRF/5 cell chemotherapy sensitivity to $\operatorname{araC}(4719.05 \mu \mathrm{M}$ in the siGAPDH group vs. $6834.30 \mu \mathrm{M}$ in the siRNA NC group) or epirubicin $(6.15 \mu \mathrm{M}$ in the siGAPDH group vs. $13.99 \mu \mathrm{M}$ in the siRNA NC group; Fig. 3A).

To investigated the underlying mechanism, the protein level of p21, which is a cyclin-dependent kinase (CDK) inhibitor, was further evaluated by Western blot analysis using the p21-specific antibody. However, no statistically significant difference was observed in the protein levels of $\mathrm{p} 21$ between cells transfected with siGAPDH or siRNA NC. In addition, p21 expression in PLC/PRF/5 cells was below the detectable levels (Fig. 3B). 
$\mathbf{A}$
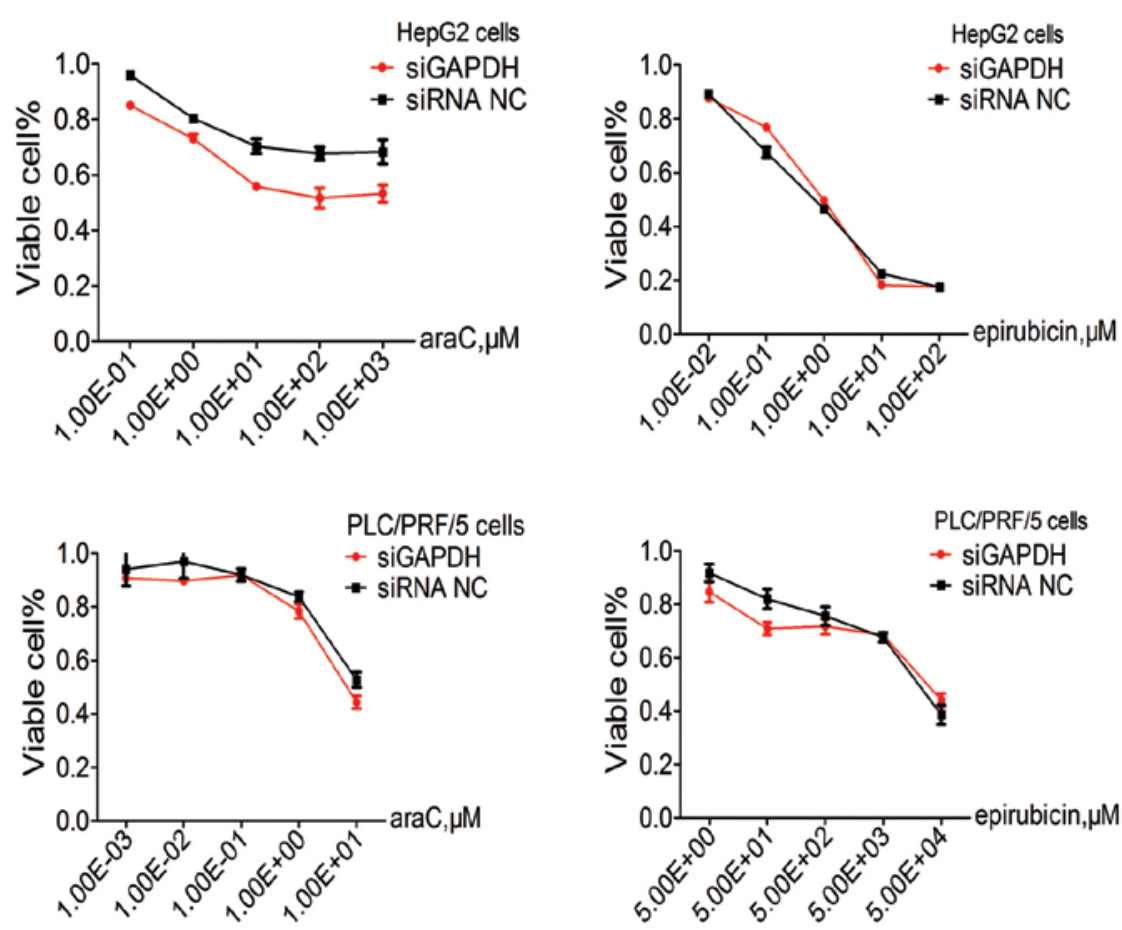

B

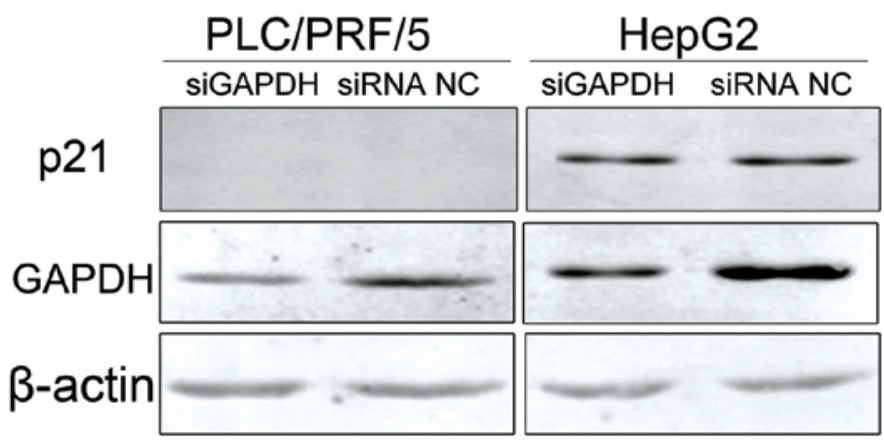

Figure 3. GAPDH expression knockdown resulted in reduced cell chemotherapy sensitivity in HepG2 cells, but not in PLC/PRF/5 cells, upon treatment with araC. (A) HepG2 cells (above) and PLC/PRF/5 cells (below) were transiently transfected with GAPDH siRNA (siGAPDH) and siRNA NC. Next, the cells were treated with araC or epirubicin and the absorbance was measured using MTT. Knockdown of GAPDH significantly alter HepG2 cells chemotherapy sensitivity to $\operatorname{araC}(\mathrm{P}=0.01)$, but did not alter cellular sensitivity to epirubicin $(10.37 \mu \mathrm{M}$ vs. $8.96 \mu \mathrm{M})$. However, GAPDH expression knockdown did not alter cellular sensitivity of PLC/PRF/5 to treatment with araC or epirubicin. The data are expressed as the mean \pm standard deviation. (B) siRNA knockdown of HepG2 and PLC/PRF/5 cells following transient transfection with GAPDH siRNA (siGAPDH) and siRNA NC. p21 protein levels were measured; however, no statistically significant differences were observed in the p21 protein levels between the siGAPDH- and siRNA NC-transfected HepG2 cells. For PLC/PRF/5 cells, the expression of p21 was not sufficiently high to detect. araC, cytosine arabinoside; GAPDH, glyceraldehyde-3-phosphate dehydrogenase.
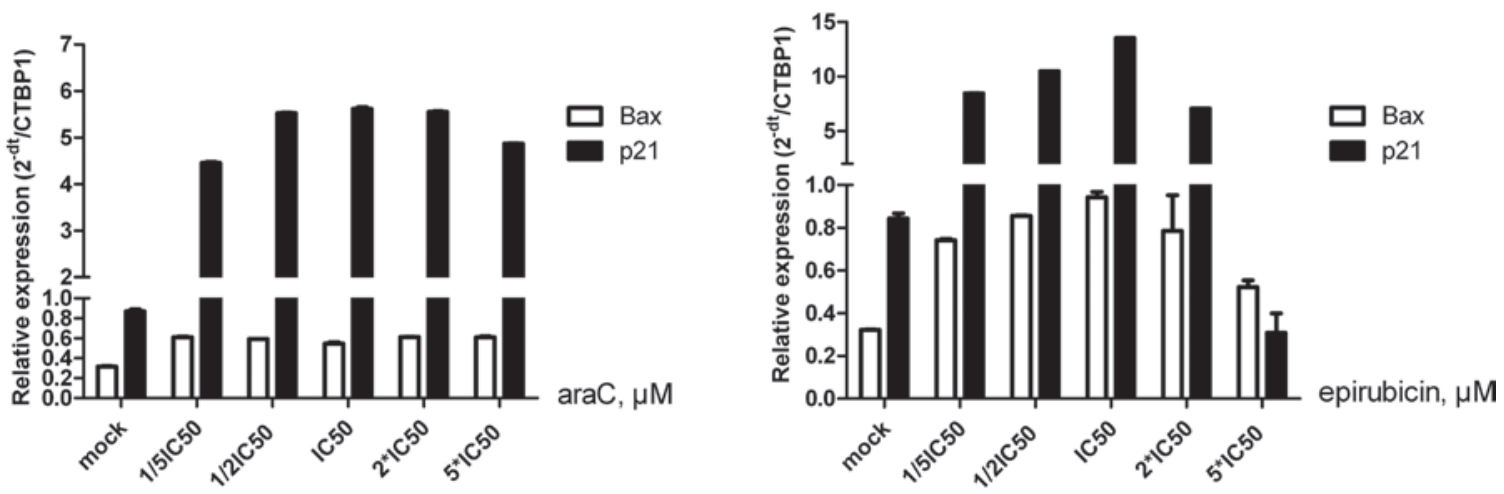

Figure 4. mRNA expression levels of p21 and Bax in HepG2 cells treated with araC or epirubicin. HepG2 cells were treated with five different concentrations of araC (left) or epirubicin (right) for $48 \mathrm{~h}$. Subsequently, the mRNA expression levels of p21 and Bax were detected using reverse transcription-quantitative polymerase chain reaction. The data represent the results of three independent experiments. Compared with the control group, the mRNA expression of p21 was increased with increasing concentrations of araC or epirubicin; however, it decreased between the doses $2 \mathrm{xIC}_{50}$ and $5 \mathrm{xIC} \mathrm{C}_{50}$. The expression of Bax increased more than twice and was slightly altered upon araC treatment, while for epirubicin, it increased about four times until the $2 \times \mathrm{XC}_{50}$ dose, and then decreased. araC, cytosine arabinoside; GAPDH, glyceraldehyde-3-phosphate dehydrogenase; Bax, B-cell lymphoma 2-associated X protein. 
A IRES2-EGFP pGAPDH-IRES2-EGFP

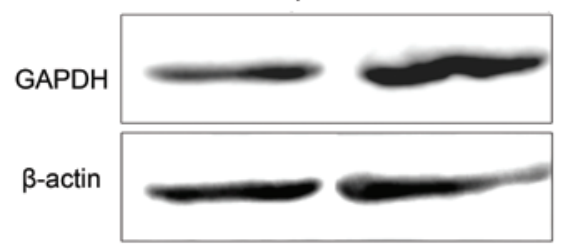

B
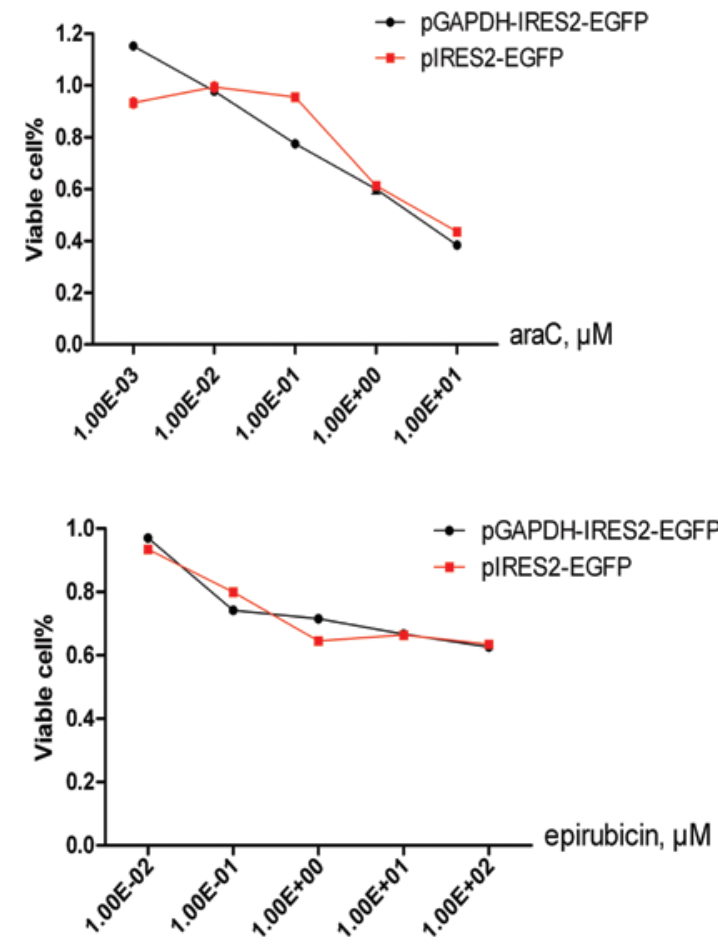

Figure 5. GAPDH overexpression did not alter HepG2 cellular sensitivity to araC and epirubicin. (A) Following transfection of HepG2 cells with GAPDH for $48 \mathrm{~h}$, GAPDH protein levels were measured using 12\% SDS-PAGE. (B) The cellular sensitivity of HepG2 cells (with overexpressed GAPDH) to araC or epirubicin was detected by MTT. Overexpression of GAPDH did not alter HepG2 cellular sensitivity to araC and epirubicin. araC, cytosine arabinoside; GAPDH, glyceraldehyde-3-phosphate dehydrogenase.

In order to investigate the effect of araC on HepG2 cellular sensitivity at the mRNA level of p21 or B-cell lymphoma 2-associated $\mathrm{X}$ protein $(\mathrm{Bax})$, the expression levels of these genes were detected following treatment with five different concentrations of araC or epirubicin for $48 \mathrm{~h}$. Compared with the control group (mock), the mRNA expression levels of p21 increased with increasing concentrations of araC or epirubicin; however, a decrease was observed between $2 \mathrm{xIC}_{50}$ and $5 \mathrm{xIC} \mathrm{IC}_{50}$ doses. By contrast, the mRNA expression of Bax following araC treatment was slightly altered. Following epirubicin treatment, the mRNA expression of Bax increased until the $\mathrm{IC}_{50}$ dose and then decreased (Fig. 4).

GAPDH overexpression did not alter HepG2 cellular sensitivity to chemotherapy with araC or epirubicin. Knockdown of GAPDH expression did not alter p21 protein level expression. Therefore, the effect of the overexpression of GAPDH on HepG2 cell sensitivity to araC or epirubicin was investigated. The HepG2 cells were initially transiently transfected with $2 \mu \mathrm{g}$ pGAPDH-IRES2-EGFP or pIRES2-EGFP and the transfection was validated by western blot analysis (Fig. 5A). Subsequently, the transfected HepG2 cells were treated with araC or epirubicin for $24 \mathrm{~h}$. As opposed to the expected result, the GAPDH overexpression was not found to alter the cellular sensitivity to $\operatorname{araC}(2.11 \mu \mathrm{M}$ vs. $2.11 \mu \mathrm{M})$ or epirubicin treatment (23.48 $\mu \mathrm{M}$ vs. $21.01 \mu \mathrm{M}$; Fig. 5B).

\section{Discussion}

Since the implementation of the national HBV vaccine immunization program in China, the overall rate of the population carrying the HBsAg declined from $9.75 \%$ in 1992 to $7.18 \%$ in 2006 (28). However, a previous study has estimated that 93 million individuals of the Chinese population are infected with chronic HBV, which may eventually result in significant public health problems in the future (29). Patients with HBV infection that is accompanied by cirrhosis are in high risk of hepatocarcinogenesis. In addition, evidence revealed that males are more prone to HCC development, compared with females (3).

GAPDH has been widely used as a reference gene in RT-qPCR analysis performed in HCC cells; however, elevated mRNA and protein expression levels of GAPDH in HCC patients have been reported in a number of studies $(30,31)$. These studies investigated patients with $\mathrm{HBV}$-associated HCC, hepatitis C virus-associated HCC and HCC without viral hepatitis infection history. Therefore, it is essential to investigate the expression of GAPDH in male patients with $\mathrm{HBV}$-associated HCC and cirrhosis.

The group of the present study has previously demonstrated the presence of aberrant GAPDH expression in male patients with HBV-associate HCC and cirrhosis (24). In addition, GAPDH expression was found to be the one of the most unstable genes among the six housekeeping gene investigated, which was consistent with a previous study on $\mathrm{HBV}$-associated HCC (32).

In the present study, $\sim 56 \%$ of tumor tissue samples exhibited differential expression of GAPDH in tumor tissues (including $37.5 \%$ of samples with increased expression and $18.1 \%$ with decreased expression) compared with non-tumor tissues, with an $>2$-fold difference. A large number of studies have demonstrated that GAPDH is involved in multiple basic cellular metabolism functions, as observed by the altered GAPDH expression levels in tumor tissues (9-12). Therefore, in the present study, HCC patients were divided into the increased and non-increased GAPDH expression groups. Notably, increased expression of GAPDH was found to be significantly associated with a lower liver fibrosis score $(\mathrm{P}=0.0394)$; to the best of our knowledge, the present study is the first to report this in male patients with HBV-associated HCC and cirrhosis. These results are in agreement with the findings of a previous study comparing healthy controls and patients with hepatitis B or $\mathrm{C}$ virus, which identified that GAPDH expression levels were significantly increased in patients with cirrhosis and the presence of HCC was closely associated with high GAPDH levels (33).

To demonstrate whether the aberrant expression of GAPDH affects the survival rates of HCC patients within a period of 60 months, survival curve analysis was performed. Notably, patients with increased GAPDH expression exhibited 
a tendency of improved survival rates; however, no statistically significant differences were observed $(\mathrm{P}=0.275)$ and, to the best of our knowledge, no similar studies has been conducted.

Numerous studies have indicated that GAPDH is involved in apoptosis and elevated GAPDH expression has been observed in HCC (17) and other cancer cells (13-16). Tumorigenesis has been hypothesized to be due to the occurrence of increased cell necrosis compared with apoptosis, which supports the observation of the present study that patients with increased GAPDH expression present higher survival rates.

Enrolling a group of patients with $\mathrm{HBV}$-associated HCC but without history of cirrhosis as the control group may have provided valuable information in the present study. However, only a few cases without cirrhosis were detected in $>500 \mathrm{HBV}$-associated HCC cases, and therefore these were not included in the present study. A similar observation was identified by Obata et al, reporting that HCC was developed only in $23 \%$ of patients who were HBsAg-positive and suffered from cirrhosis and in only $5.9 \%$ of patients who were HBsAg-negative and presented liver cirrhosis (34).

Although several studies have indicated that GAPDH is involved in chemotherapy-induced DNA damage response, the specific mechanism of GAPDH in HCC chemotherapy treatment remains unclear $(18,19)$. araC is considered to inhibit the proliferation of cells through incorporation into the DNA during replication (35), by inhabiting the DNA synthesis and arresting cell division; however, it does not disturb the RNA synthesis (36). Previous results have demonstrated that araC-induced apoptosis of cerebellar granule cells involves the expression of GAPDH and p53, while, similar to Bax, GAPDH is upregulated by p53 following exposure to the apoptotic insult (37).

In the experiments of the current study, knockdown of GAPDH expression significantly altered the sensitivity of p53-proficient HepG2 cells to araC chemotherapy, but not to epirubicin chemotherapy. However, the knockdown of GAPDH expression in the p53 mutation of PLC/PRF/5 cells did not alter the cellular chemotherapy sensitivity to araC or epirubicin. This is supported by the results of previous studies, which identified that araC-induced apoptosis was p53-dependent (38). In the present study, upon transfection of HepG2 cells with siGAPDH, the cell proliferation arrest in GAPDH-depleted cells occurred through the p53-induced expression of $\mathrm{p} 21$. In order to investigate the specific mechanism through which the GAPDH expression knockdown in HepG2 cells induced resistance to araC treatment, the protein levels of CDK inhibitor, p21waf1 cip1, were further investigated in the two cell lines. The expression of p21waf1/cip1 in $\mathrm{PLC} / \mathrm{PRF} / 5$ cells was found to be undetectable at the protein level; therefore, its effect on chemotherapy in PLC/PRF/5 cells was not evaluated.

The DNA synthesis inhibitor epirubicin eliminates cancer cells mainly via inducing G2/M arrest and apoptosis $(39,40)$. The results of the present study demonstrated that, in epirubicin treated HepG2 cells, significant upregulation of the mRNA expression levels of cell cycle progression inhibitor, p21waf1/cip1, and pro-apoptosis Bax was detected (Fig. 4). However, in araC-treated HepG2 cell, the expression of Bax was slightly altered, while the expression of p21waf1/cip1 was significantly upregulated. These differences were also observed in Fig. 3A, where epirubicin treatment significantly higher HepG2 cell apoptosis with increasing concentration.

In conclusion, to the best of our knowledge, this study is the first to report that elevated GAPDH expression in tumor tissues may be involved in the development of fibrosi. In addition, a tendency towards higher survival rates was observed for male patients with $\mathrm{HBV}$-associated HCC patients and cirrhosis; however, the underlying mechanism remains unclear. Furthermore, increased GAPDH expression may enhance the sensitivity of HCC cells to antimetabolite chemotherapy.

\section{Acknowledgements}

This study was supported by a grant from the Project for the Major Infectious Diseases from the Ministry of Science and Technology of the People's Republic of China (no. 2012ZX10002005).

\section{References}

1. Parkin DM: The global health burden of infection-associated cancers in the year 2002. Int J Cancer 118: 3030-3044, 2006.

2. He J, Gu D, Wu X, et al: Major causes of death among men and women in China. N Engl J Med 353: 1124-1134, 2005.

3. El-Serag HB and Rudolph KL: Hepatocellular carcinoma: epidemiology and molecular carcinogenesis. Gastroenterology 132: 2557-2576, 2007.

4. Ganem D and Prince AM: Hepatitis B virus infection-natural history and clinical consequences. N Engl J Med 350: 1118-1129, 2004.

5. Harada K, Hirohara J, Ueno Y, et al: Incidence of and risk factors for hepatocellular carcinoma in primary biliary cirrhosis: national data from japan. Hepatology 57: 1942-1949, 2013.

6. Lee MH, Yang HI, Liu J, et al: Prediction models of long-term cirrhosis and hepatocellular carcinoma risk risk in chronic hepatitis B patients: risk scores integrating host and virus profiles. Hepatology 58: 546-554, 2013.

7. Yang HI, Sherman M, Su J, et al: Nomograms for risk of hepatocellular carcinoma in patients with chronic hepatitis B virus infection. J Clin Oncol 28: 2437-2444, 2010.

8. Matsumura M, Ijichi M, Shiratori Y, et al: Simple quantitative assay of $\alpha$-fetoprotein mRNA in liver tissue using the real-time detection polymerase chain reaction assay - its application for clinical use. Hepatol Res 20: 84-96, 2001.

9. Sirover MA: New insights into an old protein: the functional diversity of mammalian glyceraldehyde-3-phosphate dehydrogenase. Biochim Biophys Acta 1432: 159-184, 1999.

10. Yi M, Schultz DE and Lemon SM: Functional significance of the interaction of hepatitis a virus RNA with glyceraldehyde 3-phosphate dehydrogenase (GAPDH): opposing effects of GAPDH and polypyrimidine tract binding protein on internal ribosome entry site function. J Virol 74: 6459-6468, 2000.

11. Lin SS, Chang SC, Wang YH, Sun CY and Chang MF: Specific interaction between the hepatitis delta virus RNA and glyceraldehyde 3-phosphate dehydrogenase: an enhancement on ribozyme catalysis. Virology 271: 46-57, 2000.

12. Berry MD and Boulton AA: Glyceraldehyde-3-phosphate dehydrogenase and apoptosis. J Neurosci Res 60: 150-154, 2000.

13. Vila MR, Nicolas A, Morote J, de I and Meseguer A: Increased glyceraldehyde-3-phosphate dehydrogenase expression in renal cell carcinoma identified by RNA-based, arbitrarily primed polymerase chain reaction. Cancer 89: 152-164, 2000.

14. Tokunaga K, Nakamura Y, Sakata K, et al: Enhanced expression of a glyceraldehyde-3-phosphate dehydrogenase gene in human lung cancers. Cancer Res 47: 5616-5619, 1987.

15. Revillion F, Pawlowski V, Hornez L and Peyrat JP: Glyceraldehyde-3-phosphate dehydrogenase gene expression in human breast cancer. Eur J Cancer 36: 1038-1042, 2000.

16. Harada N, Yasunaga R, Higashimura Y, et al: Glyceraldehyde3-phosphate dehydrogenase enhances transcriptional activity of androgen receptor in prostate cancer cells. J Biol Chem 282: 22651-22661, 2007. 
17. Ikeguchi M, Hirooka Y and Kaibara N: Quantitative analysis of apoptosis-related gene expression in hepatocellular carcinoma. Cancer 95: 1938-1945, 2002.

18. Meyer-Siegler K, Mauro DJ, Seal G, et al: A human nuclear uracil DNA glycosylase is the 37-KDA subunit of glyceraldehyde-3-phosphate dehydrogenase. Proc Natl Acad Sci USA 88: 8460-8464, 1991.

19. Azam S, Jouvet N, Jilani A, et al: Human glyceraldehyde3-phosphate dehydrogenase plays a direct role in reactivating oxidized forms of the dna repair enzyme ape1. J Biol Chem 283 : 30632-30641, 2008.

20. Phadke MS, Krynetskaia NF, Mishra AK and Krynetskiy E: Glyceraldehyde 3-phosphate dehydrogenase depletion induces cell cycle arrest and resistance to antimetabolites in human carcinoma cell lines. J Pharmacol Exp Ther 331: 77-86, 2009.

21. Krynetski EY, Krynetskaia NF, Gallo AE, Murti KG and Evans WE: A novel protein complex distinct from mismatch repair binds thioguanylated dna. Mol Pharmacol 59: 367-374, 2001.

22. Liu S, Zhu P, Zhang L, et al: Selection of reference genes for RT-qPCR analysis in tumor tissues from male hepatocellular carcinoma patients with hepatitis B infection and cirrhosis. Cancer Biomark 13: 345-349, 2013.

23. Heid CA, Stevens J, Livak KJ and Williams PM: Real time quantitative pcr. Genome Res 6: 986-994, 1996.

24. Gibson UE, Heid CA and Williams PM: A novel method for real time quantitative rt-pcr. Genome Res 6: 995-1001, 1996.

25. Knodell RG, Ishak KG, Black WC, et al: Formulation and application of a numerical scoring system for assessing histological activity in asymptomatic chronic active hepatitis. Hepatology 1 : 431-435, 1981.

26. Claverie JM: Computational methods for the identification of differential and coordinated gene expression. Hum Mol Genet 8: 1821-1832, 1999.

27. Xie Q, Chen X, Lu F, et al: Aberrant expression of microrna 155 may accelerate cell proliferation by targeting sex-determining region y box 6 in hepatocellular carcinoma. Cancer 118 2431-2442, 2012

28. Liang X, Bi S, Yang W, et al: Epidemiological serosurvey of hepatitis B in China - declining hbv prevalence due to hepatitis B vaccination. Vaccine 27: 6550-6557, 2009.
29. Lu FM, Li T, Liu S and Zhuang H: Epidemiology and prevention of hepatitis B virus infection in China. $\mathrm{j}$ viral hepat 17 (Suppl 1): 4-9, 2010.

30. Gong Y, Cui L and Minuk GY: Comparison of glyceraldehyde-3-phosphate dehydrogenase and 28s-ribosomal rna gene expression in human hepatocellular carcinoma. Hepatology 23: 734-737, 1996.

31. Sun W, Xing B, Sun Y, et al: Proteome analysis of hepatocellular carcinoma by two-dimensional difference gel electrophoresis: novel protein markers in hepatocellular carcinoma tissues. Mol Cell Proteomics 6: 1798-1808, 2007.

32. Fu LY, Jia HL, Dong QZ, et al: Suitable reference genes for real-time pcr in human hbv-related hepatocellular carcinoma with different clinical prognoses. BMC Cancer 9: 49, 2009.

33. Shibuya A and Ikewaki N: High serum glyceraldehyde-3-phosphate dehydrogenase levels in patients with liver cirrhosis. Hepatol Res 22: 174-179, 2002.

34. Obata H, Hayashi N, Motoike Y, et al: A prospective study on the development of hepatocellular carcinoma from liver cirrhosis with persistent hepatitis B virus infection. Int J Cancer 25: 741-747, 1980.

35. Hedley DW and McCulloch EA: Generation of reactive oxygen intermediates after treatment of blasts of acute myeloblastic leukemia with cytosine arabinoside: role of bcl-2. Leukemia 10: 1143-1149, 1996.

36. Karon M, Henry P, Weissman S and Meyer C: The effect of 1-beta-d-arabinofuranosylcytosine on macromolecular synthesis in kb spinner cultures. Cancer Res 26: 166-171, 1966.

37. Chen RW, Saunders PA, Wei H, et al: Involvement of glyceraldehyde-3-phosphate dehydrogenase (GAPDH) and p53 in neuronal apoptosis: evidence that GAPDH is upregulated by $\mathrm{p} 53$. J Neurosci 19: 9654-9662, 1999.

38. Anderson $\mathrm{CN}$ and Tolkovsky AM: A role for MAPK/ERK in sympathetic neuron survival: protection against a $\mathrm{p} 53$-dependent, JNK-independent induction of apoptosis by cytosine arabinoside. J Neurosci 19: 664-673, 1999.

39. Essmann F, Wieder T, Otto A, et al: Gdp dissociation inhibitor D4-GDI (rho-GDI 2), but not the homologous rho-GDI 1, is cleaved by caspase-3 during drug-induced apoptosis. Biochem J 346: 777-783, 2000.

40. Sun WL, Chen J, Wang YP and Zheng H: Autophagy protects breast cancer cells from epirubicin-induced apoptosis and facilitates epirubicin-resistance development. Autophagy 7: 1035-1044, 2011. 